A. Brancelj, L. De Meester \& P. Spaak (eds), Cladocera: The Biology of Model Organisms.

(C)1997 Kluwer Academic Publishers. Printed in Belgium.

\title{
Hybridization in the Daphnia galeata complex: are hybrids locally produced?
}

\author{
Piet Spaak \\ Max-Planck-Institut für Limnologie, Postfach 165, D-24302 Plön, Germany and EAWAG / ETH, Department of \\ Limnology, Ueberlandstrasse 133, 8600 Dübendorf, Switzerland (Permanent address) \\ (e-mailspaak@eawag.ch)
}

Key words: genetic distances, cladocera, allozymes, genetic diversity

\begin{abstract}
Within the species complex of Daphnia galeata, D. cucullata and D. hyalina various combinations of hybrids and parental taxa occur in lakes throughout Europe. Since daphnids are cyclic parthenogens and mostly reproduce asexually, hybrid populations can be maintained by asexual reproduction and without recurrent hybridization events. Therefore, it is possible that hybridization events have been rare, with range expansion occurring by dispersal of hybrids.

Allozyme data from seven European populations were used to compare genetic variation within and between hybrid and parental taxa. An UPGMA cluster analysis of genetic distances showed that $D$. cucullata $\times$ galeata hybrids from different lakes grouped in different clusters according to the lake from which they were isolated, suggesting multiple hybridization events. Clonal diversity within hybrid taxa was comparable to parental taxa. Furthermore, evidence was found for introgression of the Pgi-S allele from D. cucullata to D. galeata in three lakes. These results indicate that multiple hybridization events within this species complex are likely, and that hybrid taxa can reproduce sexually.
\end{abstract}

\section{Introduction}

Interspecific hybridization is a common phenomenon in Daphnia. Hybrids occur in several species complexes in North America (Colbourne \& Hebert, 1996; Hebert \& Finston, 1996), Australia (Hebert \& Wilson, 1994) and Europe (Schwenk \& Spaak, 1995). The wide distribution of Daphnia hybrids may be caused by their reproductive mode. Since Daphnia reproduce through cyclic parthenogenesis, only one or a few hybridization events are needed to establish a population of hybrids. Since these hybrids can reproduce asexually, no further hybridization events are necessary to maintain a hybrid population; presuming that environmental conditions are such that hybrids may remain present throughout the year. However, hybrid taxa also produce males and sexual females (Carvalho \& Wolf, 1989; Spaak, 1995). This creates the possibility of producing a myriad of backcrosses with parental and other hybrid taxa. Recent studies (Taylor \& Hebert, 1993; Spaak, 1996) have shown that backcrossing may be a more common process than previously believed (Wolf, 1987; Hebert et al., 1989).

Several genetic studies have shown clearly that the production of Daphnia hybrids is a continuing process (Taylor \& Hebert, 1992; Müller \& Seitz, 1995; Spaak, 1996). One argument to support this notion is that genetic variation within hybrids is comparable, or even higher, than within parental species. This would not be expected in cases where hybrids were formed a long time ago and populations persist only as parthenogenetic females. In that case, the only source for genetic variation would be the initially available variation supplemented by the variation added via mutations. As mutations also add variation to parental species, they offer an unlikely explanation for the relatively high genetic variability within hybrid populations. Furthermore, at least one hybrid was found to hatch from ephippia collected from the bottom of the Shösee in Northern Germany (Carvalho \& Wolf, 1989), and 
Schwenk (pers. comm.) recently succeeded in producing hybrids from laboratory crosses. However, these observations do not exclude the possibility that a substantial part of the hybrid lineages may be old.

One of the questions concerning hybridization in Daphnia is whether hybridizing taxa are separate species. Based on the 'Biological species concept' (Mayr, 1942) of genetically isolated taxa, one could argue that they are not if hybrids are regularly produced and if back-crossing occurs. On the other hand, several genetic studies (Taylor \& Hebert, 1992; Spaak, 1996; Schwenk, 1997) have shown that Daphnia species that produce interspecific hybrids have unique combinations of alleles, suggesting some reproductive isolation among these groups. Furthermore, parental species are morphologically distinct from each other (Flößner, 1993) and not all possible intermediate forms are observed as expected in a randomly mating 'hybrid swarm'.

More evidence for local production of interspecific hybrids in Daphnia can be obtained through comparisons of the genetic structure of the hybrid populations from isolated lakes. Let us assume that hybrids are evolutionary old, dispersed from one or a few hybrid 'sources', and that no additional hybrids or backcrosses were produced. In this scenario, one would expect hybrid populations to be characterised by: (i) a lower number of clones compared to parental taxa; (ii) a lower genetic differentiation among populations of different lakes than among hybrids and parental taxa within lakes. If hybridization is a local phenomenon, however, one would expect relative large genetic distances among hybrid taxa from different lakes. In the present study, I use two allozyme loci to compare Daphnia populations of the Daphnia galeata species complex (D. galeata, D. cucullata, D. hyalina and all three of their possible hybrids) from seven European lakes. Genetic distances among taxa within and between lakes were compared using the unweighed pair-group (UPGMA) clustering algorithm. I tested the hypothesis that hybrid populations from different lakes will cluster with the parental taxa from the same lakes rather than with the hybrids of other lakes. Support for this hypothesis indicates local and likely recurrent hybridization events in the genus Daphnia.

\section{Materials and methods}

Data on the genetic structure of seven Daphnia populations were used in this study (Table 1). Tjeukemeer is a shallow eutrophic lake in the northern part of The Netherlands, Maarsseveen is a mesotrophic lake in the centre of The Netherlands, Ringsjön is a eutrophic shallow lake situated in southern Sweden, Plußsee, Belauersee and Höftsee are situated in northern Germany, and the Bodensee is a large, deep mesotrophic lake in southern Germany. From all lakes except Ringsjön only September and October samples were used: Lake Tjeukemeer was sampled in 1989, 1990 and 1991, Maarsseveen in 1989 and 1990, Belauersee and Höftsee in 1994 and Plußsee in 1994 and 1995. Ringsjön was sampled on 16 June and 14 October 1994 by Eva Bergstrand. Data for the Bodensee were taken from Weider \& Stich (1992); I limited the analysis of this data set to only September and October sampling dates. For sampling methods and dates in Tjeukemeer see Spaak (1994; 1996); the other lakes were sampled using vertical plankton net tows (see Spaak \& Hoekstra, 1993).

Individual adult females were picked randomly and assayed using cellulose acetate electrophoresis following standard methods (Hebert \& Beaton, 1989). Generally, 100 animals per sampling date were assayed for three enzyme loci: phosphoglucomutase (Pgm, EC 5.4.2.2), phosphoglucose isomerase (Pgi, EC 5.3.1.9) and glutamate oxaloacetate transaminase (Got, EC 2.6.1.1). Got is a diagnostic marker for discrimination between $D$. galeata, D. cucullata and D. hyalina (Wolf \& Mort, 1986). D. galeata is fixed for the $F$ (fast) allele, $D$. cucullata for the $S^{-}$(very slow) allele, and $D$. hyalina for the $S$ (slow) allele. Interspecific hybrids can be identified as heterozygotes $\left(S^{-} F, S F, S^{-} S\right)$ at $\operatorname{Got}(D$. galeata $\times$ hyalina $[G \times H]: S F ; D$. cucullata $\times$ hyalina $[C \times H]: S^{-} S ; D$. cucullata $\times$ galeata $[C \times G]: S^{-} F$ ). Variation at the Pgi and Pgm loci made it possible to identify a number of two-locus genotypes within each taxon.

\section{Clonal diversity}

Clonal diversity was calculated for all taxa in all populations. As a measure of clonal diversity, I used Simpson's (1949) index of concentration, $C=\Sigma p_{i}^{2}$, where $p_{i}$ is the frequency of the $i$ th clone (twolocus genotype) in the sample, and the summation is over all clones. Clonal diversity $D$ was calculated as $D=-\log C$ (Pielou, 1975). This diversity index is a composite of abundance and evenness: it exhibits low values when a single clone is overwhelmingly predominant and high values when the number of clones is high and when clones tend to be equally abundant. 
Table 1. Average frequencies of the different taxa of the Daphnia galeata species complex for the seven studied lakes. $\mathrm{N}$ is to the total number of Daphnia individuals analysed

\begin{tabular}{llrllllll}
\hline Lake & Country & $\mathrm{N}$ & D. cucullata & D. cuc. $\times$ gal. & D. galeata & D. gal. $\times$ hyl. & D. hyalina & D. cuc. $\times$ hyl. \\
\hline Tjeukemeer & Netherlands & 1184 & 0.30 & 0.38 & 0.32 & & \\
Maarsseveen & Netherlands & 316 & & & 0.14 & 0.86 & 0.16 & 0.14 \\
Plußsee & North Germany & 743 & 0.36 & 0.20 & 0.15 & 0.13 \\
Belauersee & North Germany & 229 & 0.82 & 0.04 & & & \\
Höftsee & North Germany & 111 & & 0.10 & 0.90 & & 0.31 \\
Ringsjön & Sweden & 275 & 0.61 & 0.11 & 0.28 & & 0.20 & \\
Bodensee $^{1}$ & South Germany & 486 & & & 0.49 & 0.20 & \\
\hline
\end{tabular}

${ }^{1}$ Data from Weider \& Stich (1992)

\section{Genetic distances}

Unbiased genetic distances (Nei, 1978) among samples of the different taxa from each lake were calculated from genotype frequencies and the relatedness of the different samples was assessed using the unweighed pair-group (UPGMA) clustering program of BIOSYS1 (Swofford \& Selander, 1981). Because the number of samples was unequal for several lakes, analyses of genetic distances were based on mean frequency data per taxon per lake combining all available sampling dates. Analyses were restricted to Pgi and Pgm (Got genotypes were used to differentiate between the taxa). Höftsee was excluded from the analysis, since only 11 $C \times G$ individuals were found, leaving only one taxon for the analysis. For the same reason, $C \times G$ from Belauersee was excluded from the analysis, but the remaining two taxa in this lake (D. cucullata and $D$. hyalina) were included.

I also calculated mean genetic distances between several categories of populations to make comparisons among several classes of taxa. A population in this analysis was defined as the individuals that belong to the same taxon (hybrid or parental) in a specific lake. Comparisons were made among parental taxa (different lakes); within parental taxa (among lakes); within hybrid taxa (among lakes); and among hybrid taxa and their parental taxa in the same lake.

\section{Results}

\section{Taxon frequencies}

Based on their Got genotypes individuals were assigned to D. galeata $(F F), D$. cucullata $\left(S^{-} S^{-}\right), D$. hyalina $(S S)$ or the interspecific hybrids: In all seven studied lakes, two or more Daphnia taxa were found (Table 1). D. cucullata, D. galeata and their interspecific hybrid were the most common taxa. In three lakes, both $D$. cucullata and D. galeata were found together with their hybrid. In Bodensee, D. galeata and $D$. hyalina were found together with their interspecific hybrid. The $G \times H$ hybrid was also found in two other lakes, co-occurring with only one of the parental species (D. galeata). The $C \times H$ hybrid was found only in the Plußsee, along with one of the parental species (D. cucullata).

\section{Allele frequencies}

The most common Pgi allele found was $M$ (Table 2) with a mean frequency of $72 \%$. Both $D$. galeata and $D$. hyalina were almost fixed for this allele (frequency $>90 \%$ ) in all but one population (the $F$ allele had a frequency of $16 \%$ in the D. galeata population in the Höftsee). In $D$. cucullata, the $M$ and $S$ alleles were found at comparable frequencies. For this species, the Ringsjön population in Sweden was an exception whith the $S^{-}$allele rather than the $M$ allele having a frequency of greater then $30 \%$. As expected, the hybrid taxa showed intermediate allele frequencies, except for $C \times G$ in the Höftsee where no $S$ allele was found. In three D. galeata populations (Maarsseveen, Höftsee, Bodensee), no Pgi-S allele was found. These were also the populations were $D$. cucullata was absent.

Four alleles were found at the Pgm locus (Table 3). The most common $M$ and $F$ alleles had an equal mean overall frequency of $40 \%$. Allele frequencies of $\mathrm{Pgm}$ were less taxon-specific than for Pgi (Table 3). The $S$ allele had the highest frequency in $D$. cucullata, whereas the $F$ allele seemed to be more frequent in D. galea$t a$ and $D$. hyalina. No Pgm- $F^{+}$allele was found in $D$. cucullata. 
Table 2. Mean allele frequencies of Pgi averaged over all sampling dates for D. cucullata, D. galeata, D. hyalina and their interspecific hybrids in seven European lakes

\begin{tabular}{|c|c|c|c|c|c|c|c|c|c|c|c|c|c|c|c|c|c|c|c|c|c|c|c|c|}
\hline \multirow[t]{2}{*}{ Lake } & \multicolumn{4}{|c|}{ D. cucullata } & \multicolumn{4}{|c|}{ D. cuc. $\times$ gal. } & \multicolumn{4}{|c|}{ D. galeata } & \multicolumn{4}{|c|}{ D. gal. $\times$ hyl. } & \multicolumn{4}{|c|}{ D. hyalina } & & \multicolumn{3}{|c|}{ D. cuc. $\times$ hyl. } \\
\hline & $\mathrm{S}^{-}$ & $\mathrm{S}$ & $\mathrm{M}$ & $\mathrm{F}$ & $\mathrm{S}^{-}$ & $\mathrm{S}$ & $\mathrm{M}$ & $\mathrm{F}$ & $\mathrm{S}^{-}$ & $\mathrm{S}$ & $\mathrm{M}$ & $\mathrm{F}$ & $\mathrm{S}^{-}$ & $\mathrm{S}$ & $\mathrm{M}$ & $\mathrm{F}$ & $\mathrm{S}^{-}$ & $\mathrm{S}$ & $\mathrm{M}$ & $\mathrm{F}$ & & $\mathrm{S}^{-}$ & $\mathrm{S}$ & $\mathrm{M}$ \\
\hline Tjeukemeer & .04 & .52 & .42 & .02 & .00 & .18 & .80 & .02 & .00 & .04 & .95 & .01 & & & & & & & & & & & & \\
\hline Maarsseveen & & & & & & & & & .00 & .00 & 1.0 & .00 & .00 & .00 & 1.0 & .00 & & & & & & & & \\
\hline Plußsee & .13 & .59 & .28 & .00 & .17 & .31 & .49 & .03 & .01 & .03 & .92 & .05 & .01 & .05 & .94 & .00 & & & & & .04 & .50 & .44 & .01 \\
\hline Belauersee & .00 & .45 & .55 & .00 & .00 & .45 & .55 & .00 & & & & & & & & & .00 & .02 & .98 & .00 & & & & \\
\hline Höftsee & & & & & .00 & .00 & .96 & .04 & .00 & .00 & .84 & .16 & & & & & & & & & & & & \\
\hline Ringsjön & .37 & .56 & .06 & .01 & .19 & .33 & .48 & .00 & .00 & .01 & .98 & .01 & & & & & & & & & & & & \\
\hline Bodensee $^{1}$ & & & & & & & & & .00 & .00 & .94 & .06 & .00 & .00 & 1.0 & .00 & .00 & .00 & 1.0 & .00 & & & & \\
\hline
\end{tabular}

${ }^{1}$ Data from Weider \& Stich (1992)

Table 3. Mean allele frequencies of Pgm averaged over all sampling dates for D. cucullata, D. galeata, D. hyalina and their interspecific hybrids in seven European lakes

\begin{tabular}{|c|c|c|c|c|c|c|c|c|c|c|c|c|c|c|c|c|c|c|c|c|c|c|c|c|}
\hline \multirow[t]{2}{*}{ Lake } & \multicolumn{4}{|c|}{ D. cucullata } & \multicolumn{4}{|c|}{ D. cuc. $\times$ gal. } & \multicolumn{4}{|c|}{ D. galeata } & \multicolumn{4}{|c|}{ D. gal. $\times$ hyl. } & \multicolumn{4}{|c|}{ D. hyalina } & \multicolumn{4}{|c|}{ D. cuc. $\times$ hyl. } \\
\hline & $\mathrm{S}$ & $\mathrm{M}$ & $\mathrm{F}$ & $\mathrm{F}^{+}$ & $\mathrm{S}$ & $\mathrm{M}$ & $\mathrm{F}$ & $\mathrm{F}^{+}$ & $S$ & $\mathrm{M}$ & $\mathrm{F}$ & $\mathrm{F}^{+}$ & $\mathrm{S}$ & $\mathrm{M}$ & $\mathrm{F}$ & $\mathrm{F}^{+}$ & $\mathrm{S}$ & $\mathrm{M}$ & $\mathrm{F}$ & $\mathrm{F}^{+}$ & $\mathrm{S}$ & $\mathrm{M}$ & $\mathrm{F}$ & $\mathrm{F}^{+}$ \\
\hline Tjeukemeer & .24 & .49 & .27 & .00 & .10 & .28 & .62 & .00 & .08 & .31 & .61 & .00 & & & & & & & & & & & & \\
\hline Maarsseveen & & & & & & & & & .18 & .33 & .49 & .00 & .06 & .33 & .61 & .00 & & & & & & & & \\
\hline Plußsee & .38 & .51 & .11 & .00 & .18 & .53 & .25 & .04 & .01 & .30 & .62 & .08 & .18 & .53 & .25 & .04 & & & & & .05 & .52 & .06 & .37 \\
\hline Belauersee & .06 & .89 & .05 & .00 & .00 & .80 & .20 & .00 & & & & & & & & & .00 & .07 & .82 & .11 & & & & \\
\hline Höftsee & & & & & .41 & .49 & .05 & .05 & .18 & .42 & .36 & .04 & & & & & & & & & & & & \\
\hline Ringsjön & .49 & .46 & .05 & .00 & .14 & .72 & .14 & .00 & .04 & .64 & .30 & .02 & & & & & & & & & & & & \\
\hline Bodensee $^{1}$ & & & & & & & & & .04 & .38 & .53 & .05 & .01 & .02 & .93 & .04 & .00 & .00 & .95 & .05 & & & & \\
\hline
\end{tabular}

${ }^{1}$ Data from Weider \& Stich (1992)

\section{Clonal diversities}

Clonal diversity for several lakes did not differ much for D. cucullata, D. galeata and the hybrid $C \times G$ (Table 4). Mean values for these taxa were all above 0.60 . Clonal diversities were much lower in the $D$. hyalina, $C \times H$ and $G \times H$ populations (mean values 0.30 or less).

\section{Genetic distances}

Mean genetic distances were highest among populations of different 'parental species' (Figure 1) and lowest within these species (0.08). An average genetic distance of 0.13 was found for different populations of a given hybrid taxon. The mean genetic distance between hybrid populations and their parental taxa from the same lake was 0.12 and not significantly different from the value obtained in the species or within hybrids comparison.

The UPGMA cluster analysis of Nei's genetic distances separated the 20 taxon-lake combinations into two main groups (Figure 2). These two groups, which I will call the D. galeata and the D. cucullata group, were separated by a distance of more than 0.67 . In the $D$. galeata group, D. hyalina and $G \times H$ from Bodensee and D. hyalina from Belauersee were separated from the rest of the group by a distance of 0.09 . The Tjeukemeer population of $C \times G$ was the only ' $D$. cucullata like' hybrid that clustered in the $D$. galeata group. The $C \times H$ from Plußsee and the two populations of $C \times G$ clustered within the $D$. cucullata group. The Ringsjön population of $D$. cucullata was quite distinct from the rest of the D. cucullata group (Figure 2).

\section{Discussion}

Individual Daphnia taxa from the D. galeata species complex, sampled from geographic distant locations in Europe, showed large similarities in allele frequencies. Within species, populations from different localities showed only minor genetic differentiation among them, with the one exception being the D. cucullata population from Lake Ringsjön, Sweden (Figure 2). This population differed from the other $D$. cucullata populations by showing a high frequency $(0.37)$ of the $P g i-S^{-}$and by the occurrence of a $P g m-S$ allele. Nev- 
Table 4. Clonal diversity $D$, calculated as the negative logarithm of Simpson's index of concentration (see text) and averaged over all sampling dates, for all taxa of the D. galeata species complex in seven European lakes

\begin{tabular}{lllllll}
\hline Taxon & Bodensee $^{1}$ & Maarsseveen & Plußsee & Ringsjön & Tjeukemeer & Belauersee \\
\hline D. cucullata & & & 1.15 & 0.95 & 1.09 & 0.70 \\
D. cuc. $\times$ gal. & & & 0.54 & 0.60 & 0.79 & \\
D. galeata & 0.61 & 0.65 & 0.61 & 0.51 & 0.65 & \\
D. gal. $\times$ hyl. & 0.10 & 0.33 & 0.46 & & & \\
D. hyalina & 0.08 & & & & & 0.24 \\
D. cuc. $\times$ hyl. & & & & 0.27 & & \\
\hline
\end{tabular}

${ }^{1}$ Data from Weider \& Stich (1992)

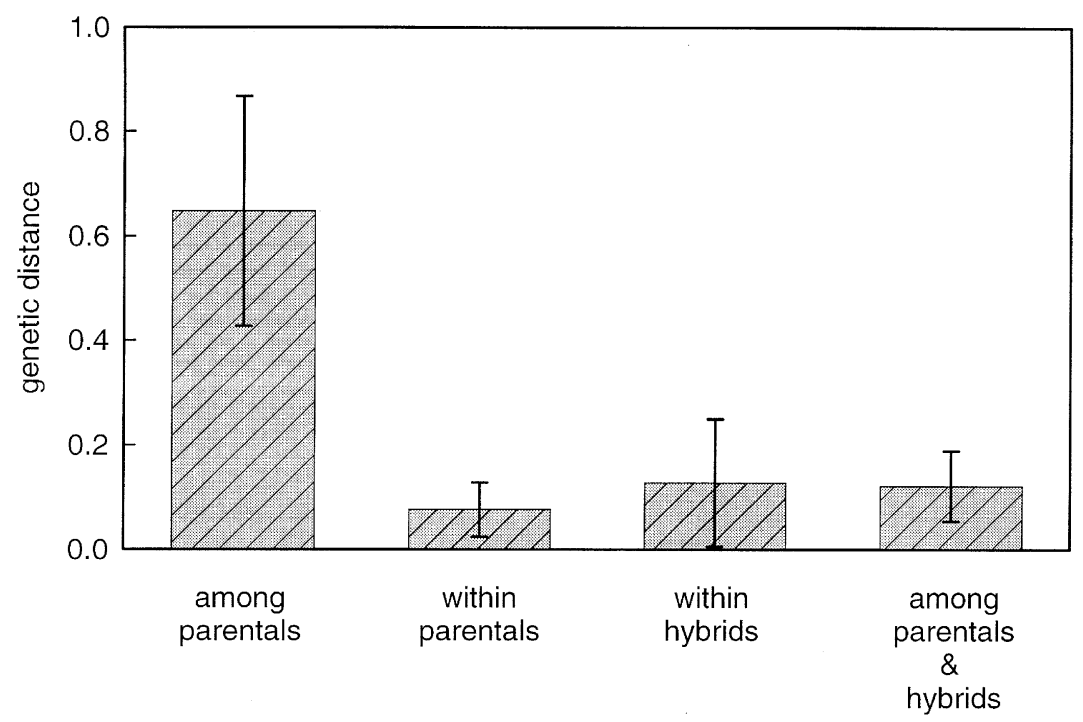

Figure 1. Mean values of Nei's genetic distance among populations of different or the same taxa. Four categories where made: among parental species (different populations), within parental species (different populations), within hybrid taxa (different populations, all hybrid taxa averaged), among parentals and hybrid taxa (within the same lake). Error bars are $95 \%$ confidence limits of the mean.

ertheless, Ringsjön $D$. cucullata still clustered within the D. cucullata group.

Recently, several studies have shown that backcrossing and introgression are important processes in Daphnia hybrid species complexes (Taylor \& Hebert, 1992; Spaak, 1996; Gießler, 1997; Schwenk, 1997). Spaak (1996) found that the population of hybrid $C \times G$ in Tjeukemeer probably consisted of a large number of backcrosses with D. galeata, and showed that $C \times G$ hybrids from Tjeukemeer clustered close to $D$. galeata. The data also suggested that the Pgi-S allele may be introgressed from $D$. cucullata to $D$. galeata through the $C \times G$ hybrid. The present study shows that the same process probably happened in Ringsjön and Plußsee. In both these lakes, D. galeata co-occurs with $D$. cucullata and their interspecific hybrid, and the $P g i-S$ allele was found in D. galeata of both lakes in low frequencies (0.01 and 0.03 respectively; Table 2), whereas this allele was not found in the three other lakes (Maarsseveen, Höftsee, Bodensee) where $D$. galeata occurred, but did not co-occur with $D$. cuculla$t a$. Despite this suggestion for backcrossing and reticulated evolution, the Plußsee and Ringsjön $C \times G$ populations clustered in the D. cucullata group (Figure 2). This suggests that other processes (e.g. differences in sexual reproduction, selection, different ages of hybrid populations) play a more important role in causing the genetic distinction between populations than the occurrence of backcrosses. 


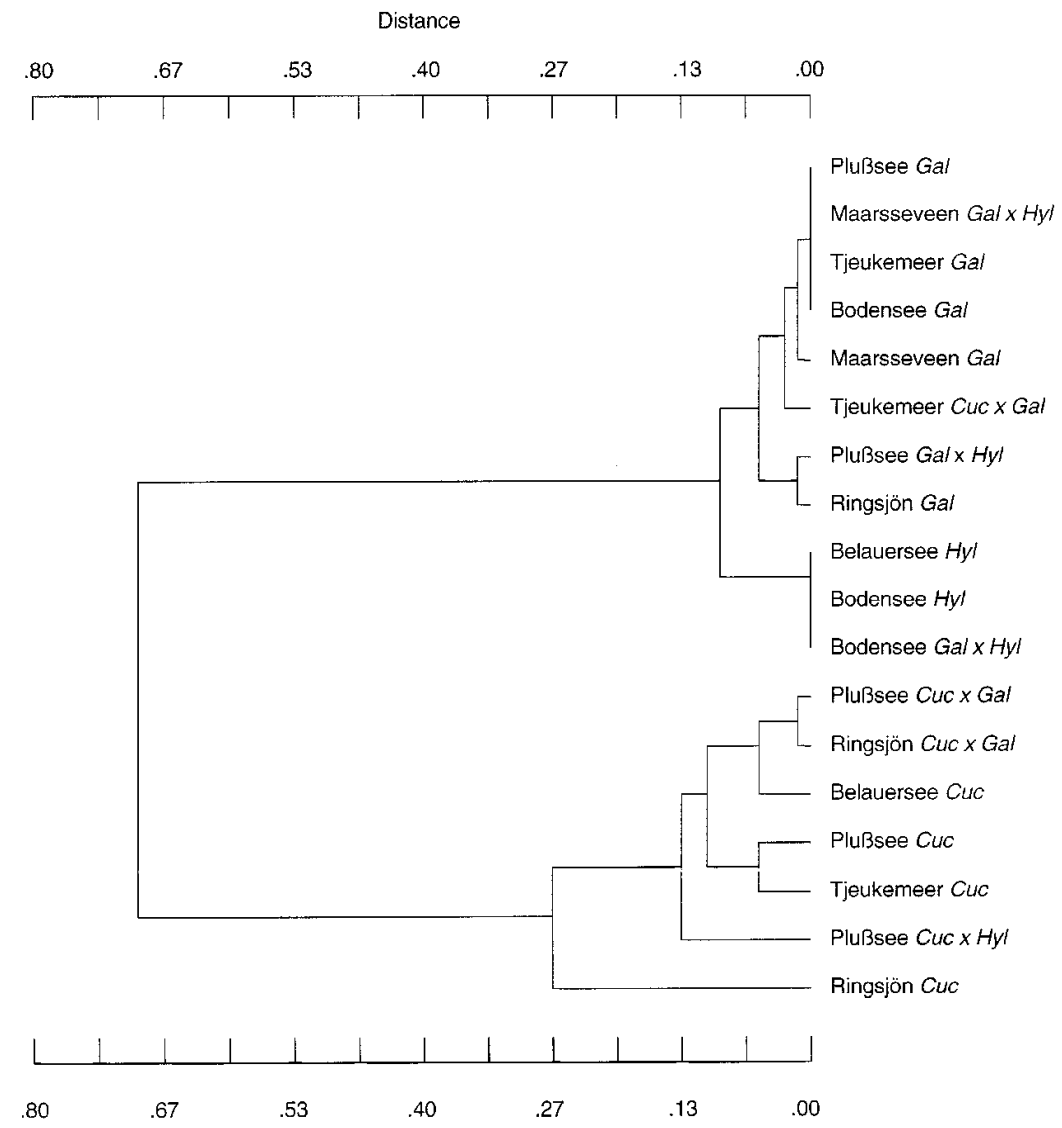

Figure 2. UPGMA clustering of Nei's genetic distance calculated for Pgi and Pgm among pooled samples of Daphnia taxa. Samples originated from six different lakes (see text for explanation).

Of the $C \times G$ hybrids, three populations were investigated. One (Tjeukemeer) clustered within the 'galeata' group, whereas the other two populations (Plußsee and Ringsjön) clustered together in the 'cucullata' group. Also, the $G \times H$ hybrids showed strong differences between populations. The Bodensee population clustered very close to $D$. hyalina, whereas the Maarsseveen and Plußsee populations clustered closer to D. galeata than to D. hyalina. These observations support the hypothesis of multiple hybridization events within the $D$. galeata species complex. If hybridization would have occurred once with the hybrids afterwards being dispersed over different European lakes, one would expect a clustering comparable to the parental species. Furthermore, if hybrids were relatively old and reproduced only parthenogenetically, one would expect a relatively low clonal diversity within hybrid populations. The observed clonal diversity, however, is in the range of the parental species. This also support the hypothesis that hybridization events still occur. Further support for this hypothesis can be derived from the mean genetic distances between taxa. In the case of 'old dispersing hybrids' one would expect a much lower differentiation within hybrid taxa compared to hybrids and parental taxa from the same lake. In fact no difference was found (Figure 1).

$C \times G$ hybrids showed a high clonal diversity in all three studied populations (Table 4), similar to the D. cucullata and D. galeata populations from the same lake. From my data it seems that sexual reproduction and hybridization events are more frequent in these taxa than in D. hyalina populations. This may be related with the type of habitats in which these taxa live (see Hrbáček, 1987). D. cucullata is a typical species of eutrophic lakes which displays high abundances in summer and autumn and disappears during winters, whereas D. galeata is frequent in lakes that freeze during winter. In contrast, D. hyalina is found in deep lakes 
where they can over-winter more easily as parthenogenetic females. This may explain the low clonal diversity within $D$. hyalina and their hybrids, although the low clonal diversity may also be caused by the low numbers of 'hyalina like' taxa found in the present study. My observations are in agreement with earlier genetic studies (Taylor \& Hebert, 1993; Müller \& Seitz, 1995) which have shown that the production of Daphnia hybrids within this species complex is most likely an ongoing process. Clearly, further experimental and field studies are needed to solve the question why hybrid and parental taxa are still morphologically and genetically distinct.

\section{Acknowledgements}

The samples of lake Ringsjön were provided by Eva Bergstrand. I thank Maarten Boersma, Luc De Meester, Jacob Müller, Klaus Schwenk, James Ward, Larry Weider, and two anonymous reviewers for their critical comments on earlier versions of this manuscript. Chris Robinson is acknowledged for correcting the English. During the German part of this study I was supported by a research fellowship from the Max Planck Society.

\section{References}

Carvalho, G. R. \& H. G. Wolf, 1989. Resting eggs of lake-Daphnia. I. Distribution, abundance and hatching of eggs collected from various depths in lake sediments. Freshwat. Biol. 22: 459-470.

Colbourne, J. K. \& P. D. N. Hebert, 1996. The systematics of NorthAmerican Daphnia (crustacea, anomopoda) - a molecular phylogenetic approach. Phil. Trans. r. Soc. Lond. B 351: 349-360.

Flößner, D., 1993. Zur Kenntnis einiger Daphnia-Hybriden. Limnologica 23: 71-79.

Gießler, S., 1997. Analysis of reticulate relationships within the Daphnia longispina species complex. Allozyme phenotype and morphology. J. Evol. Biol. 10: 87-105.

Hebert, P. D. N. \& M. J. Beaton, 1989. Methodologies for allozyme analysis using cellulose acetate electrophoresis. Helena laboratories Beaumont, Texas, $32 \mathrm{pp}$.

Hebert, P. D. N. \& T. L. Finston, 1996. A taxonomic reevaluation of North-American Daphnia (Crustacea, Cladocera). 2. New species in the Daphnia-pulex group from the South-Central United-States and Mexico. Can. J. Zool. 74: 632-653.
Hebert, P. D. N., S. S. Schwartz \& J. Hrbáček, 1989. Patterns of genotypic diversity in Czechoslovakian Daphnia. Heredity 62: 207-216.

Hebert, P. D. N. \& C. C. Wilson, 1994. Provincialism in plankton: endemism and allopatric speciation in Australian Daphnia. Evolution 48: 1333-1349.

Hrbáček, J., 1987. Systematics and biogeography of Daphnia species in the northern temperate regions. In R. H. Peters \& R. de Bernardi (eds), Daphnia, Pallanza, Mem. Inst. ital. Idrobiol. 45: 37-76.

Mayr, E., 1942. Systematics and the origin of species. Columbia University Press, New York, 332 pp.

Müller, J. \& A. Seitz, 1995. Differences in genetic structure and ecological diversity between parental forms and hybrids in a Daphnia species complex. Hydrobiologia 307: 25-32.

Nei, M., 1978. Estimation of average heterozygosity and genetic distance from a small number of individuals. Genetics 89: 583 590.

Pielou, E. C., 1975. Ecological Diversity. Wiley-Interscience, New York, $165 \mathrm{pp}$.

Schwenk, K., 1997. Evolutionary genetics of Daphnia species complexes -hybridism in syntopy. Ph.D Thesis, University of Utrecht, The Netherlands, $141 \mathrm{pp}$.

Schwenk, K. \& P. Spaak, 1995. Evolutionary and ecological consequences of interspecific hybridization in cladocerans. Experientia 51: 465-481.

Simpson, E. H., 1949. Measurement of diversity. Nature 163: 688.

Spaak, P., 1994. Genetical ecology of a coexisting Daphnia hybrid species complex. Ph.D Thesis, University of Utrecht, The Netherlands, $125 \mathrm{pp}$.

Spaak, P., 1995. Sexual reproduction in Daphnia: interspecific differences in a hybrid species complex. Oecologia 104: 501-507.

Spaak, P., 1996. Temporal changes in the genetic structure of the Daphnia species complex in Tjeukemeer, with evidence for backcrossing. Heredity 76: 539-548.

Spaak, P. \& J. R. Hoekstra, 1993. Clonal structure of the Daphnia population in Lake Maarsseveen: its implications for diel vertical migration. Arch. Hydrobiol. Beih. Ergebn. Limnol. 39: 157-165.

Swofford, D. L. \& R. B. Selander, 1981. BIOSYS-1: a fortran program for the comprehensive analysis of electrophoretic data in population genetics and systematics. J. Hered. 72: 281-283.

Taylor, D. J. \& P. D. N. Hebert, 1992. Daphnia galeata mendotae as a cryptic species complex with interspecific hybrids. Limnol. Oceanogr. 37: 658-665.

Taylor, D. J. \& P. D. N. Hebert, 1993. Habitat-dependent hybrid parentage and differential introgression between neighboringly sympatric Daphnia species. Proc. Natl. Acad. Sci. USA. 90: 7079-7083.

Weider, L. J. \& H. B. Stich, 1992. Spatial and temporal heterogeneity of Daphnia in Lake Constance; intra- and interspecific comparisons. Limnol. Oceanogr. 37: 1327-1334.

Wolf, H. G., 1987. Interspecific hybridization between Daphnia hyalina, D. galeata and D. cucullata and seasonal abundance of these species and their hybrids. Hydrobiologia 145: 213-217.

Wolf, H. G. \& M. A. Mort, 1986. Interspecific hybridization underlies phenotypic variability in Daphnia populations. Oecologia 68: 507-511. 